\title{
A New Wrist Clinical Evaluation Score
}

\author{
Guillaume Herzberg, MD, $\mathrm{PhD}^{1}$ Marion Burnier, MD ${ }^{1}$ Toshiyasu Nakamura, MD, $\mathrm{PhD}^{2}$ \\ ${ }^{1}$ Hôpital Edouard Herriot, Lyon, France \\ ${ }^{2}$ Clinical Research Center, International University of Health and \\ Welfare, Tokyo, Japan
Address for correspondence Guillaume Herzberg, MD, PhD, Hôpital Edouard Herriot, 5 Place d'Arsonval, Lyon 69003, France \\ (e-mail: guillaume.herzberg@chu-lyon.fr).
}

J Wrist Surg 2018;7:109-114.

\begin{abstract}
Keywords

- clinical score

- wrist

- evaluation

- wrist surgery

- clinical

- outcomes
\end{abstract}

An optimal assessment of the clinical status and outcomes of treatment is a critical part of any clinical osteoarticular research.

Historically, the clinical assessment of osteoarticular wrist conditions was first performed with physician-based scoring systems. ${ }^{1-5}$ More recently, patient-rated outcome measures (PROMs), ${ }^{6}$ such as Quick Dash and patient-rated wrist evaluation (PRWE), have been designed. These are widely used for the evaluation of wrist conditions. The number of physician-based wrist-scoring systems that are currently available is limited. ${ }^{1-4}$ All of them are very old and some of them do not include forearm rotation criteria. Not

received

August 10, 2017

accepted

September 12, 2017

published online

October 30, 2017 including the latter criteria may induce some bias (i.e., missing an important part of the symptoms) in the clinical evaluation of wrist conditions, since forearm rotation is an important part of wrist capabilities. This is particularly true when evaluating the clinical results of distal radius fractures.

Moreover, we are not aware of any electronically automated wrist-scoring systems.

The purpose of this article was (1) to describe a new electronic wrist clinical score, the Lyon wrist score (LWS) and (2) to present a new patient's generated wrist evaluation criterion, the subjective wrist value (SWV).
Copyright $\odot 2018$ by Thieme Medical Publishers, Inc., 333 Seventh Avenue, New York, NY 10001, USA. Tel: +1(212) 584-4662.
DOI https://doi.org/ 10.1055/s-0037-1607328. ISSN 2163-3916. 


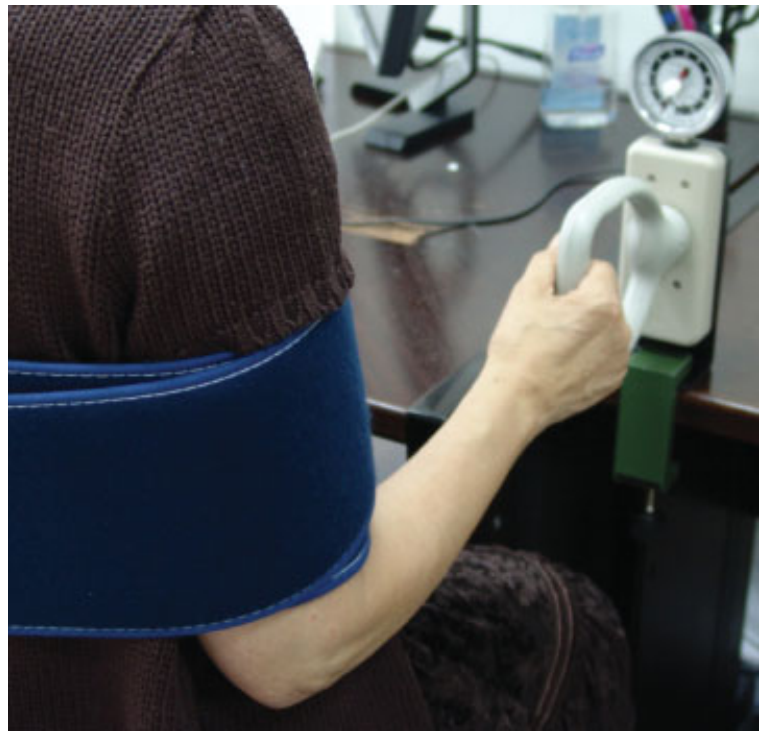

Fig. 1 Measurement of the forearm rotational strength with a light mobile device (baseline wrist dynamometer, Fabrication Enterprises Inc., Elmsford, NY) during clinical visits.

Both LWS and SWV are combined into a single graphical display providing a comprehensive display of osteoarticular wrist clinical status.

\section{Materials and Methods}

The electronic Lyon wrist score (LWS) is an Excel file that includes four classic wrist clinical criteria: pain, function, active motion, and strength. Each criterion is given a numerical value among five classes (from normal to maximally disabled: 20-15-10-5-0). Normal pain, function, active motion, and strength are rated as 20 points each, i.e., a sum of 80 which is then expressed as percentage to define a total numerical score. Each criterion is split into numerically equivalent subcriteria. This allows a separate evaluation of wrist pain and function when the patient performs flexion-extension (which reflects radio- and midcarpal joints status) or forearm rotation (which reflects distal radio-ulnar joint provided that the more proximal components of the forearm are intact).

Both pain and function are evaluated on a visual analog scale (VAS) according to patient's real-life activities whether these are just activities of daily living, work, or sports-related activities.

Motion and strength are first entered as numerical values, respectively, in degrees and kilograms, then automatically converted into sums (for motion) and percentages (for strength), which in turn allows to choose scores among five class values. Radial and ulnar deviation values are recorded into the chart but not included into the standard/full LWS score calculations. Regarding motion and strength, the contralateral values must be filled out.

Measurement of forearm pronation and supination normal strength (matched to age and gender) with a light mobile device $\boldsymbol{- F i g .} 1$ has recently been published. ${ }^{7,8}$ If the physi- cian's office is equipped with a forearm rotational strength measurement light mobile device (baseline wrist dynamometer, Fabrication Enterprises Inc., Elmsford, NY), the LWS can include the forearm rotational strength values.

Because forearm rotation strength measurement devices are not widely spread yet, we designed a "standard LWS" including only the grip strength and a "full LWS" including both grip and forearm rotation strength.

The total (or full) LWS scores are provided as percentage values with higher scores indicating a better result.

The graph also includes a new clinical wrist parameter, the subjective wrist value (SWV). The SWV is a new single numeric percentage value that can be easily generated and reported at each patient visit. It is exactly similar to the SSV, subjective shoulder value, ${ }^{9}$ which has a widespread use in shoulder surgery. The SWV was determined entirely subjectively by each patient, who answered the following question: "What is the overall percent value of your wrist if a completely normal wrist represents $100 \%$ ?" If a patient was unclear about the question or desired further information, the explanation consisted of the following standardized statement and question: "A completely normal wrist would cost you 1,000 euros. How much would you be willing to pay for yours?"

If the LWS displays a postoperative clinical status, the subjective result is included at the bottom of the chart.

Regardless of the standard or full version of the LWS, the physician will have to fill out exclusively the dark gray numeric case boxes (-Figs. 2 and 3). The light gray numeric cases are automatically generated calculations. These must be kept intact as calculation boxes.

Although the data do not necessarily have to be entered in a standardized sequential manner, we recommend to do so as shown in - Figs. 4 and $\mathbf{5}$. A total of 23 dark gray excel boxes must be filled out in the standard wrist form, whereas 28 dark gray excel boxes must be filled out in the full wrist form. If the opposite wrist is abnormal, motion and strength reference values ${ }^{10,11}$ are provided into the form, respectively, in degrees and kilograms.

\section{Results}

The total standard (or full) LWS numerical scores were automatically generated. Four LWS classes were arbitrarily defined as excellent (equal or superior to $90 \%$ ), good (equal or superior to $70 \%$ and inferior to $90 \%$ ), fair (equal or superior to $50 \%$ and inferior to $70 \%$ ), and poor (inferior to $50 \%$ ). These LWS classes may be used as pre- and/or postoperative clinical evaluations.

The standard (or full) LWS components were automatically included into a diamond-shaped graph that included the SWV as well. The diamond-shaped graph is a practical tool, which may be copied and pasted as such into a PowerPoint presentations for demonstration and comparison purposes.

The complete standard and full LWS charts are displayed in -Figs. 2 and 3, respectively. Both standard and full LWS charts are available for free, downloadable in colored versions at alcoms69@lyon.fr. 


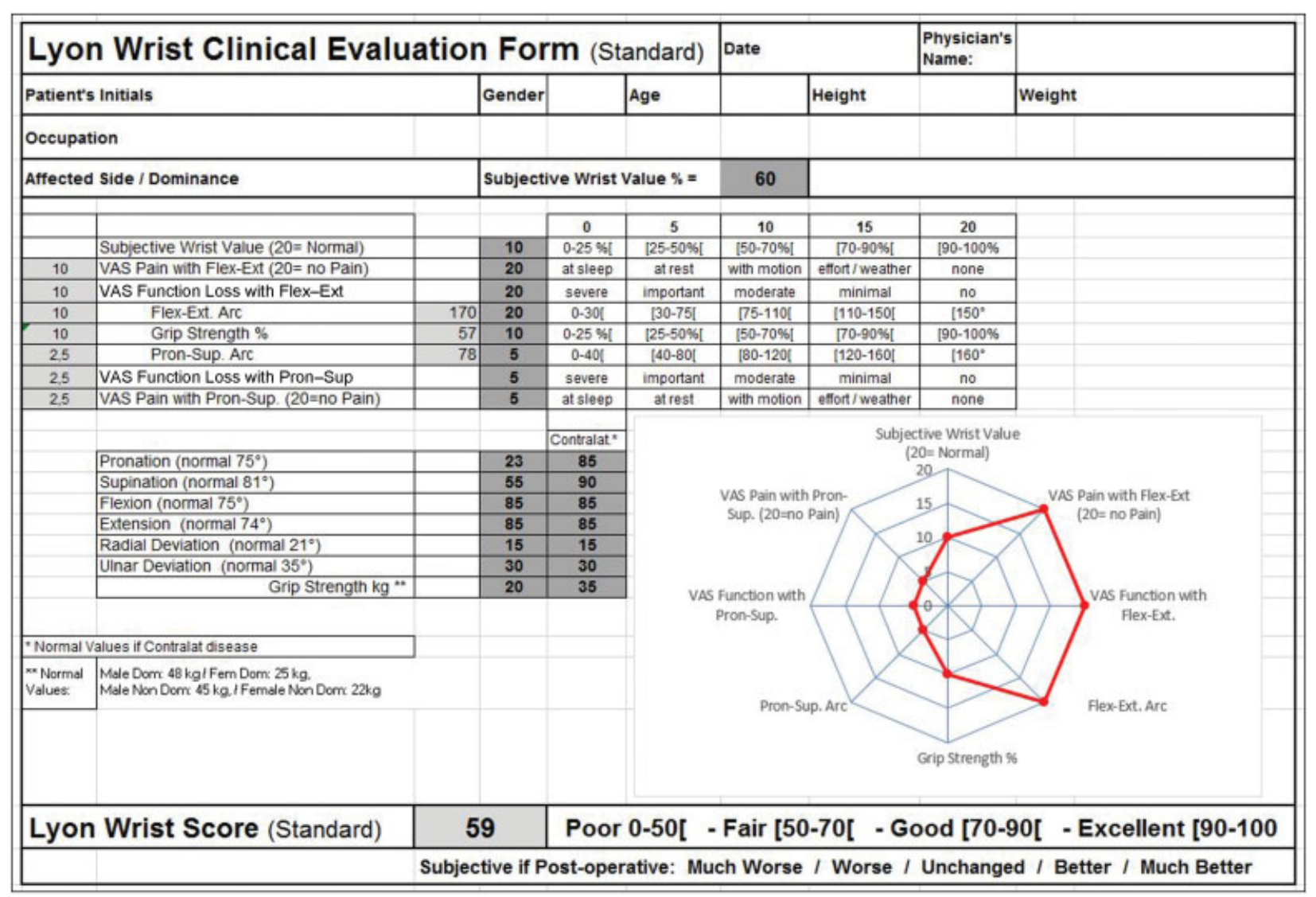

Fig. 2 Standard LWS chart. The physician filled out exclusively the 23-dark gray numeric case boxes. The light gray boxes are automatic calculation boxes and should be left undisturbed. Here is shown an imaginary example of a wrist with marked distal radioulnar joint impairment. LWS, Lyon wrist score.

\section{Discussion}

The use of clinical scores in wrist surgery is mandatory to compare pre- and postoperative status, which in turn is an essential step to define the usefulness of a surgical procedure. The important attributes of a clinical outcome score are that it should accurately reflect the perspective of the patient, include the physician's classic criteria, and should be independent of the diagnosis. The first wrist clinical scores were used to combine clinical and radiological criteria. ${ }^{3-5}$ More recent wrist scores were purely clinical, ${ }^{1,2}$ which is logical, since clinical and radiological outcomes often diverge. However, these were not available in electronic files, and some of them did not include forearm rotation criteria.

In this article, the authors present a comprehensive userfriendly new clinical wrist score (LWS) based on the four classic clinical criteria (VAS pain, VAS function, motion, and strength), including forearm rotation, VAS pain, function, and motion. The authors also present a new simple subjective patient-related criteria (SWV). Both LWS and the SWV can be gathered into a graph, which may be used both on a routine clinic basis and/or for research and presentation/comparison purposes.

Regarding the LWS, we thought it was important to separately analyze wrist radiocarpal criteria (VAS radiocarpal pain, VAS radiocarpal function, active wrist flexion-extension, grip strength) and forearm rotation criteria (VAS distal radioulnar joint [DRUJ] pain, VAS DRUJ function, active forearm rotation, grip strength). The advantage of evaluating function on a VAS is that function is tailored to patient's real-life activities, whether these are just activities of daily living, work, or sports-related activities.

Measurement of grip strength with a Jamar device is widely accepted. ${ }^{1,2,12,13}$ A Jamar dynamometer is available nowadays in any wrist focused unit. Measurement of forearm rotation strength, which should reflect the DRUJ status provided that the rest of the forearm is normal, should be its counterpart but it is not often used so far. The use of a mobile device for forearm rotation measurement - Fig. 1 is currently uncommon but may become more widespread in the future. Light mobile devices for forearm rotational strength measurement at clinics are available in the market. ${ }^{8,14}$ It is the author's opinion that evaluation of the forearm rotational strength should be part of wrist evaluation in the future. For this reason, the LWS is available in standard version (without forearm rotation strength evaluation) for those physicians who do not have a mobile device for forearm rotation measurement. A full version includes the forearm rotation strength evaluation.

The LWS is open in that each main criterion may be split into more subcriteria if necessary in the future. For example, ulnar-radial inclinations or a wrist three-dimensional evaluation of dart-throwing motion may be included in the future as a new subcriterion. 
112 New Wrist Clinical Evaluation Score Herzberg et al.

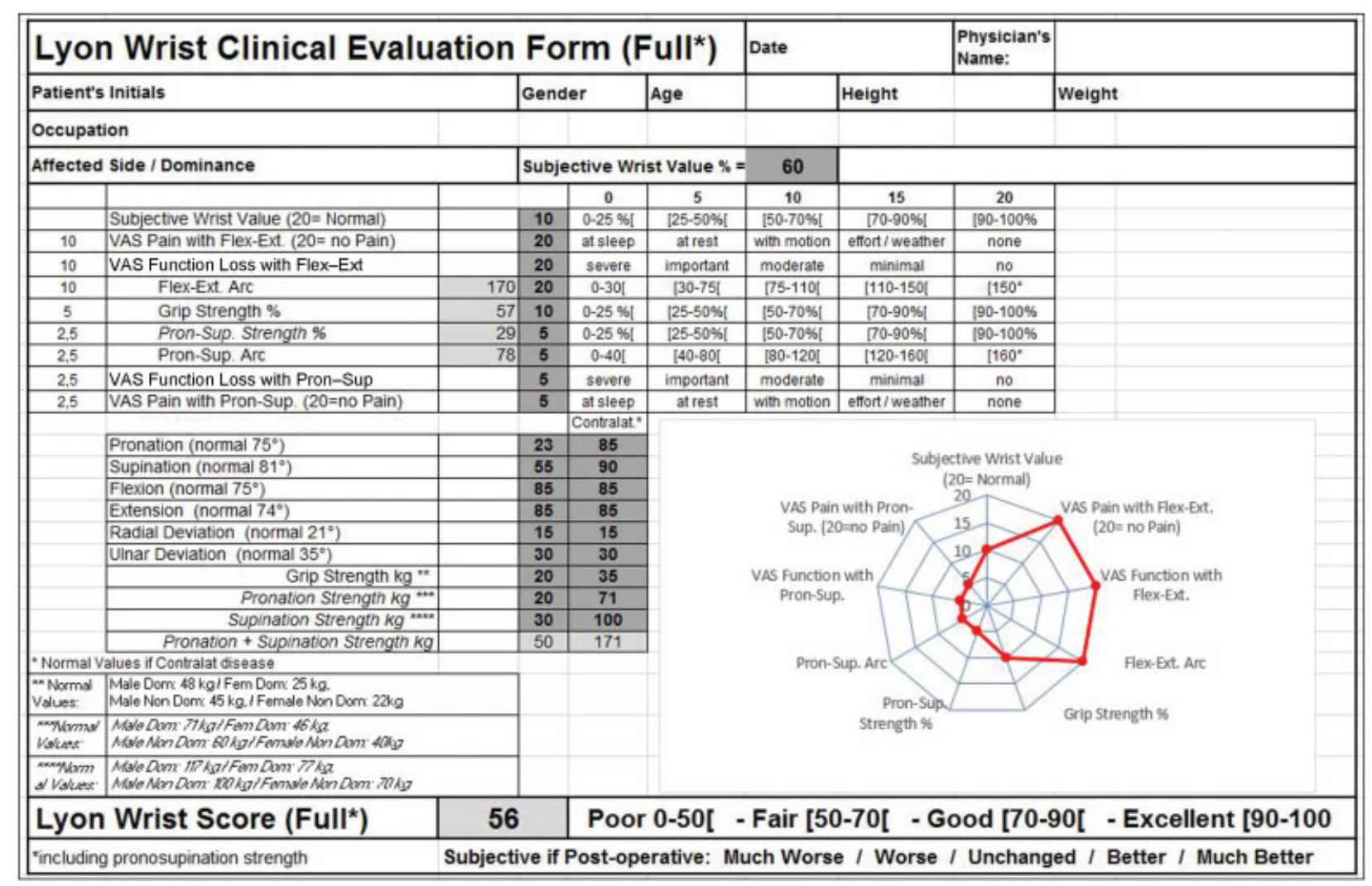

Fig. 3 Full LWS chart. The physician filled out exclusively the 28-dark gray numeric case boxes. The light gray boxes are automatic calculation boxes and should be left undisturbed. Here is shown an imaginary example of a wrist with marked distal radioulnar joint impairment (same values as - Fig. 2 chart with the addition of forearm rotation strength). LWS, Lyon wrist score.

\begin{tabular}{|c|c|c|c|c|c|c|c|}
\hline \multicolumn{4}{|c|}{ Lyon Wrist Clinical Evaluation Form (Standard) } & \multicolumn{2}{|l|}{ Date } & \multirow[t]{2}{*}{\begin{tabular}{|l|} 
Physician's \\
Name:
\end{tabular}} & \multirow[b]{2}{*}{ Weight } \\
\hline Patient's Initials & Gender & & Age & & Height & & \\
\hline \multicolumn{8}{|l|}{ Occupation } \\
\hline Affected Side / Dominance & \multicolumn{3}{|c|}{ Subjective Wrist Value $\%=$} & 1 & & & \\
\hline & & 0 & 5 & 10 & 15 & 20 & \\
\hline Subjective Wrist Value $(20=$ Normal) & 2 & $0-25 \%[$ & [25-50\%[ & {$[50-70 \%[$} & $770-90 \%[$ & {$[90-100 \%$} & \\
\hline VAS Pain with Flex-Ext ( $20=$ no Pain) & 3 & at sleep & at rest & with motion & effort/weather & none & \\
\hline VAS Function Loss with Flex-Ext & 4 & severe & important & moderate & minimal & no & \\
\hline Flex-Ext. Arc & 21 & $0-30[$ & {$[30-75[$} & {$[75-110[$} & {$[110-150[$} & {$\left[150^{*}\right.$} & \\
\hline Grip Strength \% & 22 & $0-25 \%[$ & [25-50\%] & {$[50-70 \%[$} & {$[70-90 \%[$} & {$[90-100 \%$} & \\
\hline Pron-Sup. Arc & 23 & $0-40[$ & {$[40-80[$} & {$[80-120[$} & {$[120-160[$} & {$\left[160^{\circ}\right.$} & \\
\hline VAS Function Loss with Pron-Sup & 5 & severe & important & moderate & minimal & no & \\
\hline VAS Pain with Pron-Sup. (20=no Pain) & 6 & at sleep & at rest & with motion & effort/ weather & none & \\
\hline & & & & & & & \\
\hline & & Contralat* ${ }^{*}$ & & & & & \\
\hline Pronation (normal $75^{\circ}$ ) & 7 & 8 & & & & & \\
\hline Supination (normal $81^{\circ}$ ) & 9 & 10 & & & & & \\
\hline Flexion (normal 75) & 11 & 12 & & & & & \\
\hline Extension (normal $74^{\circ}$ ) & 13 & 14 & & & & & \\
\hline Radial Deviation (normal $21^{\circ}$ ) & 15 & 16 & & & & & \\
\hline UInar Deviation (normal $35^{\circ}$ ) & 17 & 18 & & & & & \\
\hline Grip Strength $\mathrm{kg}$ ** & 19 & 20 & & & & & \\
\hline
\end{tabular}

Fig. 4 Standard LWS chart sequential fill-out sequence. Although the data do not necessarily have to be entered in a standardized sequential manner, this figure shows the most logical and efficient sequential fill-out sequence ( 23 dark gray boxes). LWS, Lyon wrist score. 


\begin{tabular}{|c|c|c|c|c|c|c|c|}
\hline \multirow{2}{*}{\begin{tabular}{|l} 
Lyon Wris \\
Patient's Initials
\end{tabular}} & & & $u \mid l *)$ & \multicolumn{2}{|l|}{ Date } & \multirow{3}{*}{\begin{tabular}{|l} 
Physician's \\
Name:
\end{tabular}} & \multirow{3}{*}{ Weight } \\
\hline & Gen & & Age & & Height & & \\
\hline Occupation & & & & & & & \\
\hline \multirow[t]{2}{*}{ Affected Side / Dominance } & \multicolumn{3}{|c|}{ Subjective Wrist Value $\%=$} & 1 & & & \\
\hline & & 0 & 5 & 10 & 15 & 20 & \\
\hline Subjective Wrist Value $(20=$ Normal) & 2 & $0-25 \%[$ & {$[25-50 \%[$} & {$[50-70 \%[$} & {$[70-90 \%[$} & {$[90-100 \%$} & \\
\hline VAS Pain with Flex-Ext. ( $20=$ no Pain) & 3 & at sleep & at rest & with motion & effort/ weather & none & \\
\hline VAS Function Loss with Flex-Ext & 4 & severe & important & moderate & minimal & no & \\
\hline Flex-Ext. Arc & 25 & $0-30[$ & {$[30-75[$} & {$[75-110[$} & {$[110-150[$} & {$\left[150^{\circ}\right.$} & \\
\hline Grip Strength \% & 26 & $0-25 \%[$ & {$[25-50 \%[$} & {$[50-70 \%[$} & {$[70-90 \%[$} & {$[90-100 \%$} & \\
\hline Pron-Sup. Strength \% & 27 & $0-25 \%[$ & {$[25-50 \%[$} & {$[50-70 \%[$} & {$[70-90 \%[$} & {$[90-100 \%$} & \\
\hline Pron-Sup. Arc & 28 & $0-40[$ & {$[40-80[$} & {$[80-120[$} & {$[120-160[$} & {$\left[160^{\circ}\right.$} & \\
\hline VAS Function Loss with Pron-Sup & 5 & severe & important & moderate & minimal & no & \\
\hline VAS Pain with Pron-Sup. ( $20=$ no Pain) & 6 & at sleep & at rest & with motion & effort / weather & none & \\
\hline & & Contralat. ${ }^{*}$ & & & & & \\
\hline Pronation (normal $75^{\circ}$ ) & 7 & 8 & & & & & \\
\hline Supination (normal $81^{\circ}$ ) & 9 & 10 & & & & & \\
\hline Flexion (normal $75^{\circ}$ ) & 11 & 12 & & & & & \\
\hline Extension (normal $74^{\circ}$ ) & 13 & 14 & & & & & \\
\hline Radial Deviation (normal $21^{\circ}$ ) & 15 & 16 & & & & & \\
\hline Ulnar Deviation (normal $35^{\circ}$ ) & 17 & 18 & & & & & \\
\hline \begin{tabular}{|c|} 
Grip Strength $\mathrm{kg}$ ** \\
\end{tabular} & 19 & 20 & & & & & \\
\hline Pronation Strength $\mathrm{kg}^{\mathrm{kn}}$ & 21 & 22 & & & & & \\
\hline Supination Strength $\mathrm{kg}$ & 23 & 24 & & & & & \\
\hline Pronation + Supination Strength $\mathrm{kg}$ & & & & & & & \\
\hline
\end{tabular}

Fig. 5 Full LWS chart sequential fill-out sequence. Although the data do not necessarily have to be entered in a standardized sequential manner, this figure shows the most logical and efficient sequential fill-out sequence (28 dark gray boxes). LWS, Lyon wrist score.

The score is designed to evaluate all osteoarticular carpal pathological conditions and/or DRUJ conditions irrespective of their posttraumatic, arthritic, or inflammatory etiology. The score is not designed to be used for assessment of osteoarticular forearm pathological conditions, such as Essex Lopresti fracture. However, it may be used to assess the outcome of Galeazzi sequelae, since the pathology is located at the radius and distal radioulnar joint.

Recently, we added to our electronic wrist evaluation chart a new patient's measure of wrist function, the subjective wrist value (SWV) by analogy with the validated subjective shoulder value (SSV), ${ }^{9}$ which is widely used in shoulder surgery. The SWV is an easily administered measure of wrist function. It is the exact reproduction of the subjective shoulder value (SSV). Both LWS and SWV are combined into a single graphical display.

This article has weaknesses, since the LWS + SWV evaluation system has only been used in our unit so far. More than 1,000 standard LWS and more than 100 full LWS were filled out so far. It has not been validated per se but it is a combination of many well-accepted criteria plus some new criteria. Inter- and intraobserver studies should be performed in the future.

There are several strengths in our article: the chart (LWS and SWV) is user friendly, quick, and easy to fill out by an assistant during physical examination by the physician. It is the first electronic wrist score, including automated calculations and providing an instant diamond-shaped graph. The graph provides a comprehensive display of any osteoarticular wrist clinical status. Not only the number of purely clinical criteria is the largest so far among all available wrist scores, but the graphic representation displays an unprecedented comprehensive representation of wrist clinical status. This may facilitate the presentations, comparisons, and discussions of clinical cases during meetings.

Conflict of Interest

None.

\section{Acknowledgments}

The authors wish to thank David Slutsky, MD, Michel Boeckstyns, MD, and Jan Ragnar Haugstvedt, MD for their assistance in the writing of this article.

\section{References}

1 Cooney WP, Bussey R, Dobyns JH, Linscheid RL. Difficult wrist fractures. Perilunate fracture-dislocations of the wrist. Clin Orthop Relat Res 1987;(214):136-147

2 Cooney WP, Linscheid RL, Dobyns JH. Triangular fibrocartilage tears. J Hand Surg Am 1994;19(01):143-154

3 Gartland JJ Jr, Werley CW. Evaluation of healed Colles' fractures. J Bone Joint Surg Am 1951;33-A(04):895-907

4 Green DP, O'Brien ET. Open reduction of carpal dislocations: indications and operative techniques. J Hand Surg Am 1978;3 (03):250-265

5 Sarmiento A, Pratt GW, Berry NC, Sinclair WF. Colles' fractures. Functional bracing in supination. J Bone Joint Surg Am 1975;57 (03):311-317

6 Weldring T, Smith SM. Patient-reported outcomes (PROs) and patient-reported outcome measures (PROMs). Health Serv Insights 2013;6:61-68

7 Herzberg G, Berthonnaud E, Bestion A, Dimnet J. Testing of pronation and supination strength in the outpatient setting: a 
114 New Wrist Clinical Evaluation Score Herzberg et al.

preliminary study [Article in French]. Chir Main 2007;26(01): 40-43

8 Rey PB, Jardin E, Uhring J, Obert L. Existe-t-il une corrélation entre la force de pronation-supination du côté dominant et celle du côté non dominant chez l'adulte sain droitier: résultats préliminaires. Chir Main 2014;33(01):17-22

9 Gilbart MK, Gerber C. Comparison of the subjective shoulder value and the Constant score. J Shoulder Elbow Surg 2007;16 (06):717-721

10 Boone DC, Azen SP. Normal range of motion of joints in male subjects. J Bone Joint Surg Am 1979;61(05):756-759
11 Greene WB. The clinical measurement of joint motion. Rosemont Illinois, IL: American Academy of Orthopaedic Surgeons; 2017

12 Bechtol CO. Grip test; the use of a dynamometer with adjustable handle spacings. J Bone Joint Surg Am 1954;36-A(04):820-824, passim

13 Lee JA, Sechachalam S. The effect of wrist position on grip endurance and grip strength. J Hand Surg Am 2016;41(10):e367-e373

14 Herzberg G, Parmentier H, Erhard L. Assessment of functional outcome in hand transplantation patients. Hand Clin 2003;19 (03):505-509 